\title{
Target Selection on Mobile Devices using Display Segmentation
}

\author{
David Dearman \\ Dept. of Computer Science \\ University of Toronto \\ Toronto, Ontario, Canada \\ dearman@cs.toronto.edu
}

\author{
Kori M. Inkpen \\ Faculty of Computer Science \\ Dalhousie University \\ Halifax, Nova Scotia, Canada \\ inkpen@cs.dal.ca
}

\author{
Khai N. Truong \\ Dept. of Computer Science \\ University of Toronto \\ Toronto, Ontario, Canada \\ khai@cs.toronto.edu
}

\begin{abstract}
In this paper, we explore the use of an interaction technique called sequential segmentation to support target selection for mobile devices. Sequential segmentation iteratively partitions an information space into selectable regions and subsequent sub-regions where each region/sub-region is labeled (1-9) and is mapped to the corresponding key on the mobile device's numeric keypad. We conducted a study comparing the sequential technique to the directional pad for target selection. The results show that the directional pad is significantly faster than sequential for selecting targets that require three or less interactions with the directional pad. However, sequential is significantly faster than the directional pad for targets that require five or more interactions with the directional pad. User feedback shows a preference for sequential and that it is perceived easier to use than the directional pad.
\end{abstract}

\section{Categories and Subject Descriptors}

H.5.2 [User Interfaces]: Interaction Styles, Evaluation/ Methodology

\section{Keywords}

Display segmentation, sequential segmentation, target selection, mobile interaction, user study

\section{INTRODUCTION}

Target selection in a mobile device application can be a non-trivial task given the limited input affordances of the device. Touch sensitive displays provide an intuitive and direct interaction with the display space and selectable targets, whereas mobile devices with only the traditional 12 button numeric keypad (1-9, *, 0 and \#) do not offer a similarly obvious nor effective method for target selection. Numerous interaction modalities have been proposed to augment the limited avenues of interaction that rely on sensors $[1,5]$, a camera [7] and appropriately mapping the keypad to the display's information space $[6,4,3]$. Although numerous

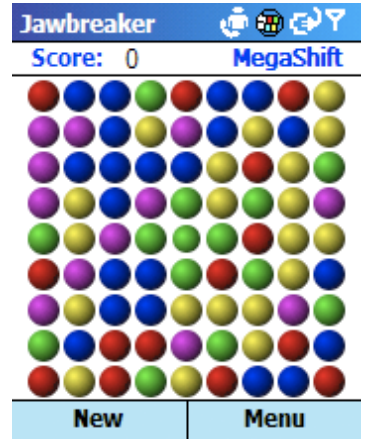

(a) Jawbreaker

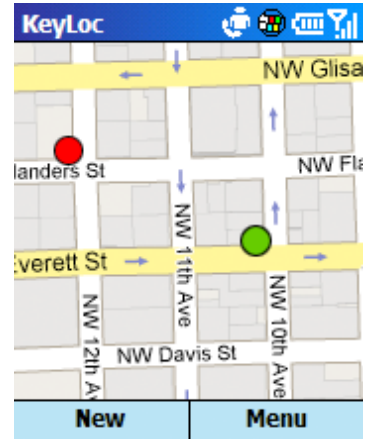

(b) Map Locations
Figure 1: Example applications for mobile devices where selection is problematic and display segmentation could be applied.

modes of interactions are possible, we focus on leveraging the limited affordance of the numeric keypad rather than augmenting the device itself.

This paper explores the use of a technique called sequential segmentation (abbreviated sequential) that iteratively segments the information space on a mobile device for target selection using the numeric keypad. Segmentation has been previously explored on mobile devices for the navigation of information space such as a map (ZoneZoom [6]) or a large data set (FaThumb [4]), exploring one-handed interactions (AppLens and LaunchTile [3]) and for text entry (TNT [2]). Each application of segmentation, although similar in design, was specific to a unique application space. FaThumb and ZoneZoom support the navigation of a hierarchical information space, while the evaluation of AppLens and LaunchTile was limited to the gesture interaction which required a touch sensitive display. Inspired by these previous works, we explore the benefit of our sequential, two-click segmentation technique for target selection utilizing only the keypad for input. The technique provides a simple twostage interaction for refinement and target selection where the information space is partitioned into selectable regions that correspond to the numeric keys on the keypad. Addressing the need for alternate and appropriate interaction techniques for mobile devices is important because of the growing application of mobile computing to a greater range of activities in varying contexts. Whether the user needs to 


\begin{tabular}{|c|c|c|}
\hline KeyLoc & \multicolumn{2}{|c|}{ 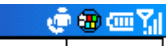 } \\
\hline 11 & 2 & (3) \\
\hline 4 & 5 & 6 \\
\hline 7 & 8 & 9 \\
\hline & & dido \\
\hline
\end{tabular}

(a) Seq. regions

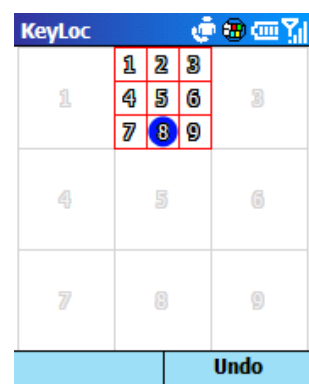

(b) Seq. sub-regions

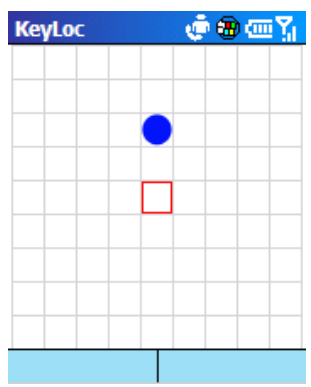

(c) D.Pad start

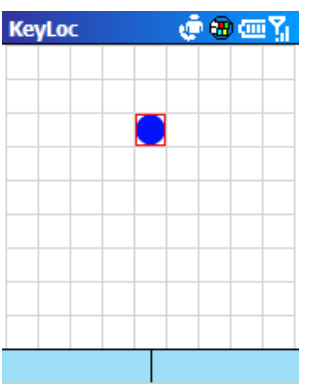

(d) D.Pad move
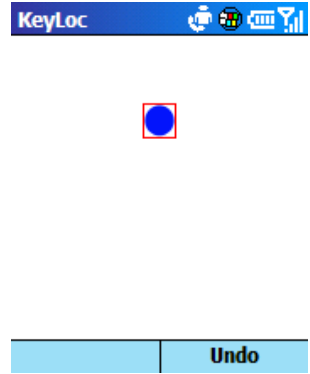

(e) Target Selected

Figure 2: Example usage of sequential (a, b, e) and the directional pad (c, d, e) to select a target. Using sequential, (a) the information space is initially segmented into nine selectable regions. Once a region is selected, (b) the region is further segmented into nine selectable sub-regions, where (e) the target can be selected. Using the directional pad, (c) the cursor starts in the center region and then is moved over the target (d) where a selection is made (e).

select a ball (Jawbreaker (Figure 1(a)) for a game, a location annotated on a location-aware mobile map (Figure 1(b)), a link on a web page or an application widget, the selection technique should be appropriate given many usages.

In the following sections, we provide an overview of sequential and our user study to evaluate sequential against the directional pad for target selection. The results of the study show that the directional pad is significantly faster than sequential for selecting targets that require three or less interactions with the directional pad and significantly slower than sequential for targets that require five or more interactions with the directional pad. Additionally, using sequential, selecting targets that extend multiple sub-regions is significantly slower than targets fully contained with a sub-region. User feedback shows a preference for sequential and that it is perceived to be easier to use than the directional pad.

\section{SEQUENTIAL SEGMENTATION}

The sequential technique is based on the process of segmenting the information space into selectable regions, similar to that used in ZoneZoom [6]. However, our technique iteratively segments the display to provide more fine-grained selection. The information space is initially segmented into nine regions, forming a $3 \times 3$ matrix, where each region is labeled 1-9 (see Figure 2(a)). The numeric label (1-9) identifies a regions mapping to the corresponding key on the keypad. Pressing a key selects the likewise labeled region. Sequential further sub-divides the selected region into another $3 \times 3$ matrix, where each sub-region is again labeled 1-9 (see Figure 2(b)). The user then presses the key corresponding to the appropriate sub-region to select a target (see Figure 2(e)). Sequential benefits from simplicity and consistency. The number of selectable regions/sub-regions is consistent (nine) and the mapping of the regions to the keypad is identical for all the regions/sub-regions. Using the circle target in Figure 1 as a reference, the user would press the 2 key followed by the 8 key to select the target.

\section{EVALUATION}

We conducted a within-subjects study to evaluate sequential segmentation against the directional pad which is typically used for navigation and selection on some mobile devices. We evaluated the techniques using a simple target selection task.

\subsection{Participants and Apparatus}

We recruited 18 right-handed university students (9 male, 9 female) as participants. The majority of participants (12) owned a mobile phone that they used on a daily (11) or semi-daily basis (1). Of the remaining six participants, five indicated less frequent usage and one had no previous mobile phone experience.

Participants performed the experiment using an Audiovox SMT5600 Smartphone running a custom application developed in C\# using the .NET compact framework.

\subsection{Experimental Design}

The study used a within-subjects design where the two techniques (the experimental conditions), sequential and the directional pad were counterbalanced. Participants completed 3 blocks of 18 selection tasks, where a blue dot measuring $19 \times 20$ pixels displayed on the SMT5600 is selected. Targets are placed at varying distances from the screen's centre and are chosen according to three target categories; full, half and quarter selectable. Full targets are completely within the bounds of a selectable sub-region, half targets straddle two neighboring sub-regions and quarter targets straddle the intersection of four neighboring sub-regions. The ordering of the targets was randomized to ensure participants could not predict the location of the next target.

To ensure a fair comparison, the information space for the directional pad we segmented into 81 equally sized regions; analogous in size to the sub-regions for sequential. A square cursor highlighted the current region and moved from region to region using the directional pads up, down, left and right. By default at the start of each selection task, the cursor was placed in the horizontal and vertical center region (see Figure 2(c)). Once participants moved the cursor over a target (see Figure 2(d)), a selection is made (see Figure 2(e)) by pressing the directional pad inwards. 


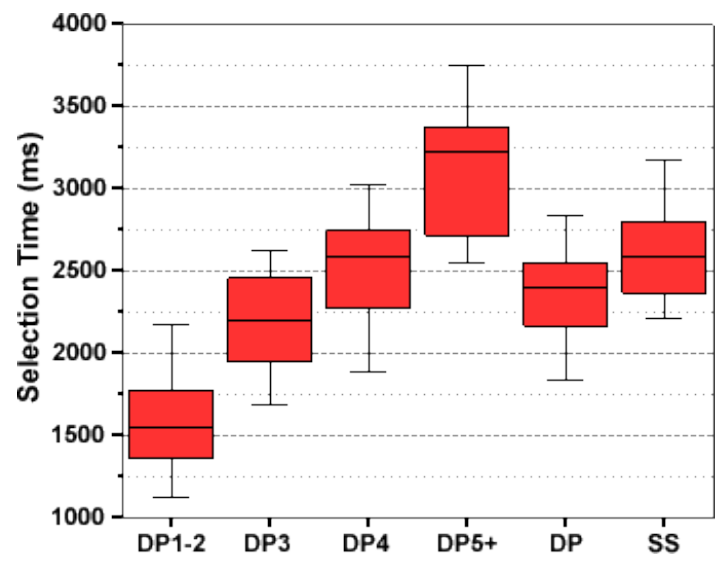

Figure 3: Box plot of target selection time for the four interaction categories (DP1-2, DP3, DP4, DP5+) using the directional pad and the overall time for the directional pad (DP) sequential (SS).

All interactions with the device were recorded by the study software, including button presses and timing data. Selections were classified as errors if the sub-region selected did not contain any portion of the target.

\subsection{Procedure}

Participants completed background questionnaires to help us assess their mobile phone experience. Next, participants completed tasks in each experimental condition. Each condition began with a short training session consisting of nine target selection tasks to familiarize the participants with the interaction technique. We then administered the experimental session consisting of 54 selection tasks, followed by a questionnaire asking the participants to provide feedback on the interaction technique used. This process was repeated for the two conditions. After the final condition, participants were given a final questionnaire asking them to reflect on both techniques.

\section{RESULTS AND DISCUSSION}

Approximately $1 \%$ of trials were identified as outliers $(>3$ SD) and were removed from the data set. Target selection times were analyzed using repeated measures ANOVA with the Huynh-Feldt correction when the sphericity assumption was violated. Post hoc pairwise comparisons are conducted using the Bonferroni adjustment.

\subsection{Selection Time}

Analysis on selection times shows that the directional pad was significantly faster than sequential $(\mathrm{F}(1,17)=10.35$, $\mathrm{p}<0.01$ ) for target selection (see Figure 3). However, this result does not tell the full story. Although the directional pad is significantly faster than sequential, the number of interactions required to select a target is an important consideration. Target selection using sequential always requires two interactions because the technique is not position dependent. The directional pad, however, is position dependent, requiring an increasing number of interactions to select a target as the distance between the start and target location increases. To analyze this effect on selection time, the

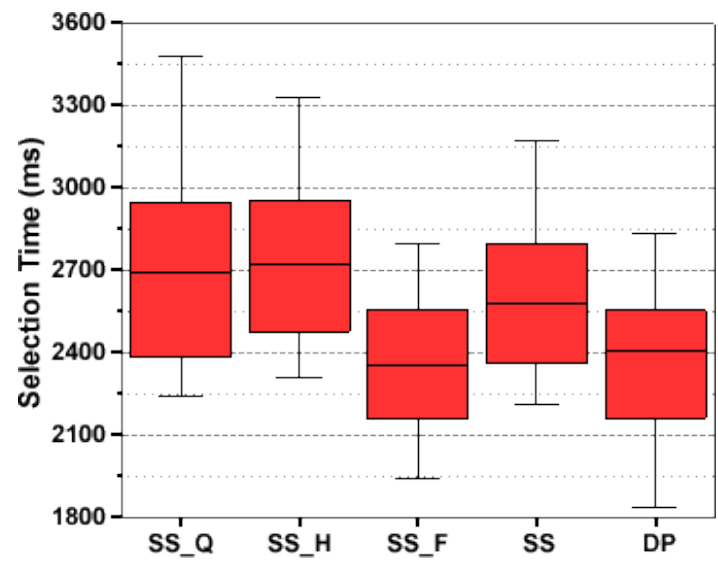

Figure 4: Box plot of target selection time for sequential and the directional pad. Additionally, target selection time for quarter (SS_Q), half (SS_H) and full (SS_F) selectable targets using sequential.

targets were divided into four categories with each category having four or five targets. The four categories are defined by the number of interactions with the directional pad that are required to navigate to and select a target: one to two, three, four, and five or more. Results (see Figure 3) show that the number of interactions (or equally the distance) for a target significantly affected selection time, $\mathrm{F}(2.89,49.16)$ $=141.9, \mathrm{p}<0.001$. Pairwise comparison shows sequential is significantly faster $(\mathrm{p}<0.001)$ than the directional pad for targets that require five or more interactions to make a selection. No significant difference $(p=1.0)$ could be shown between sequential and the directional pad for targets that required four interactions to make a selection.

The mean selection time for targets that require four interactions is statistically equivalent to the two interactions required by sequential. This is interesting because it suggests that the cognitive demand associated with mapping the information space to the keypad for sequential plays an important role in the selection time. The cognitive demand associated with the movements for the directional pad is arguably low, as such the directional pad is faster for targets requiring one to three interactions to make a selection. However, as the distance between the starting region and the target region grows beyond four interaction, the directional pad become increasingly slower because of the higher number of interactions required to navigate the information space. Sequential requires only two interactions regardless of the target's location.

\subsection{Target Categories}

Analysis of the three target categories (full, half and quarter) shows that the target position within a sub-region significantly influenced $(\mathrm{F}(2,34)=64.66, \mathrm{p}<0.001)$ target selection time for sequential (see Figure 4). Pairwise comparison revealed that full targets are significantly faster $(\mathrm{p}<0.001)$ to select than half and quarter targets. No significant difference $(p=1.0)$ was shown for the selection time of half and quarter targets. This result suggests that selecting targets that straddle multiple sub-regions requires greater cognitive 
Table 1: The median value of the rank ( $1=$ preferred) and Likert scale (1-Strongly Disagree; 5Strongly Agree) questionnaire responses.

\begin{tabular}{|l|c|c|c|c|c|}
\hline & & \multicolumn{4}{|c|}{ Ease of } \\
& Pref. & \multicolumn{2}{|c|}{ Understanding } & \multicolumn{2}{c|}{ Use } \\
\hline & Rank & Rank & Scale & Rank & Scale \\
\hline SS & 1 & 2 & 5 & 1 & 5 \\
\hline DP & 2 & 1 & 5 & 2 & 4 \\
\hline p & $<0.01$ & $=0.059$ & $=0.018$ & $<0.05$ & $<0.05$ \\
\hline
\end{tabular}

demand when using sequential. Targets that straddle multiple sub-regions (half and quarter) require the user to make an additional decision for which sub-region to select given multiple possibilities. Targets full within a sub-region do not incur this additional overhead as there is only one possible choice. This is an important design consideration that needs to be investigated as it is unlikely targets will fit perfectly within a defined sub-region in an unstructured application.

\subsection{Selection Errors}

A selection error was identified as a target selection that did not include any portion of the target. A total of 43 errors were identified from the 1944 target selections ( $\sim 98 \%$ overall accuracy). There were 37 error for the directional pad and six for sequential. We believe that the greater number of error incurred by the directional pad can be attributed to the SMT5600 hardware itself. Participants commented that the directional pad sometimes registered selections when the participant's intent was a directional movement.

\subsection{Questionnaire Data}

Upon completion of each technique, participants were asked to rate the technique on a five point Likert scale (1-Strongly Disagree to 5-Strongly Agree) on perceived ease of use and ease of understanding. Upon completing the final session, participants were also asked to rank the two techniques in terms of preference, ease of use and ease of understanding (see Table 1). The rank and Likert scale data was analyzed using Friedman's Two-Way Analysis of Variance. Analysis revealed that participants perceived sequential to be significantly easier to use than the directional pad (rank: $\chi^{2}=$ $5.56, \mathrm{p}<0.05$; scale: $\chi^{2}=6.40, \mathrm{p}<0.05$ ) and no significant difference for the techniques ease of understanding (rank: $\chi^{2}$ $=3.56, \mathrm{p}=0.059$; scale: $\left.\chi^{2}=1.80, \mathrm{p}=0.18\right)$. This is important because it suggests that participants did not perceive the mapping between the information space and the keypad more demanding than navigation using the directional pad. Finally, participants indicated that for target selection they significant preferred sequential (rank: $\chi^{2}=8.00, \mathrm{p}<0.01$ ) over the directional pad.

\section{CONCLUSION AND FUTURE WORK}

Target selection on input constrained mobile devices is difficult unless the device provides a mode for direct interaction such as a touch sensitive display. In this paper, we outline a technique called sequential segmentation that iteratively segments the information space to provide target selection using the numeric keypad. We then evaluated sequential against the directional pad. Results show that the directional pad is significantly faster than sequential for selecting targets that require three or less interactions with the directional pad and significantly slower than sequential for targets that require five or more interactions with the directional pad. The directional pad shows a benefit for proximally close targets, but becomes increasingly slower as the distance and the number of interactions required to select a target grows. The significant benefit for distant targets when using sequential is attributed to its consistent two click interaction. Questionnaire feedback shows a preference for sequential and that it is perceived to be easier to use than the directional pad.

In future work, the selection of overlapping or proximal targets needs to be explored. Currently we assume only one target is within a sub-region, however, in practice this is unlikely to be the case. Additionally, we are looking at alternate mappings between segmented regions and the keypad.

\section{ACKNOWLEDGMENTS}

The authors would like thank all the participants for their time. This research is supported in part by the Natural Science and Engineering Research Council of Canada (NSERC) and the Walter C. Sumner Foundation. This work is covered under the Research Ethics Board of Dalhousie University, protocol \#2006-1341.

\section{REFERENCES}

[1] K. Hinckley, J. Pierce, M. Sinclair, and E. Horvitz. Sensing techniques for mobile interaction. In UIST '00: Proceedings of the 13th annual ACM symposium on User interface software and technology, pages 91-100. ACM Press, 2000.

[2] M. Ingmarsson, D. Dinka, and S. Zhai. Tnt: a numeric keypad based text input method. In $\mathrm{CHI}$ '04: Proceedings of the SIGCHI conference on Human factors in computing systems, pages 639-646. ACM Press, 2004.

[3] A. K. Karlson, B. B. Bederson, and J. SanGiovanni. Applens and launchtile: two designs for one-handed thumb use on small devices. In CHI '05: Proceedings of the SIGCHI conference on Human factors in computing systems, pages 201-210. ACM Press, 2005.

[4] A. K. Karlson, G. G. Robertson, D. C. Robbins, M. P. Czerwinski, and G. R. Smith. Fathumb: a facet-based interface for mobile search. In CHI '06: Proceedings of the SIGCHI conference on Human Factors in computing systems, pages 711-720. ACM Press, 2006.

[5] J. Rekimoto. Tilting operations for small screen interfaces. In UIST '96: Proceedings of the 9th annual ACM symposium on User interface software and technology, pages 167-168. ACM Press, 1996.

[6] D. C. Robbins, E. Cutrell, R. Sarin, and E. Horvitz. Zonezoom: map navigation for smartphones with recursive view segmentation. In $A V I$ '04: Proceedings of the working conference on Advanced visual interfaces, pages 231-234. ACM Press, 2004.

[7] M. Sohn and G. Lee. Iseeu: camera-based user interface for a handheld computer. In MobileHCI '05:

Proceedings of the 7th international conference on Human computer interaction with mobile devices 8 services, pages 299-302. ACM Press, 2005. 